\title{
Notes on Meteorological Observations at Plymouth.
}

\author{
By
}

\section{H. N. Dickson, F.R.S.E.}

The meteorological observations at Plymouth Navigation School, carried on for a period of more than twenty-six years by Dr. J. Merrifield, ceased on his death in June last. It seemed desirable that Plymouth should not be without an observing station, and as the Association was undertaking other work of a similar nature it was decided to begin observations on the scale of a station of the second order.

Mr. W. V. Merrifield, into whose hands the instruments passed on his father's death, kindly offered to lend a set of thermometers and sunshine recorder. The barometer used by Dr. Merrifield is the property of the Meteorological Council, who have transferred the instrument temporarily to the hands of the Association until another instrument can be acquired.

The ground behind the Laboratory, being almost enclosed by the walls of the Citadel, was obviously unsuitable for the exposure of meteorological instruments. Application was accordingly made to the municipal authorities of Plymouth for permission to erect a thermometer screen and to expose a rain gauge on the enclosed ground behind the public lavatory on the Hoe. This permission was granted by the Hoe Committee, and an exceptionally good exposure has been obtained close to the Laboratory.

In order to make the observations as generally useful as possible, copies are regularly supplied to the following:-(1) The Meteorological Office, weekly and monthly Reports.

(2) The Royal Meteorological Society, monthly Reports. (3) The Medical Officer of Health, fortnightly Reports. (4) Western Morning News, daily Report and monthly Summary.

The sheets are issued from the Laboratory with all corrections made, and summed and averaged. The following is a list of the elements observed and calculated :-Atmospheric pressure. Temperature: Dry bulb, Wet bulb, Maximum, Minimum, Dew-point. -Pressure of vapour-Relative humidity. Wind : Direction, Force 
(Beaufort Scale). Cloud: Form, Amount (0-10). Rainfall. Sunshine. Ozone (0-10).

The barometer is of marine pattern (B. T. 59), reading to .002 in., and hangs in an unused room with a north light. The height of the cistern above mean sea level at Devonport Dockyard is 125.9 feet, as ascertained by levels from Ordnance datum mark, executed by Plymouth Borough Engineer. The reduction of observations to sea level is effected by tables specially supplied by the Meteorological Office.

The thermometers are exposed in a Stevenson screen of the usual pattern, open below. A complete duplicate set of thermometers is kept in readiness in case of accident. Observations are made with Negretti and Zambra's ozone tests, the papers being hung in the Stevenson screen.

The rain gauge is of Meteorological Office pattern, with circular rim 8 inches in diameter. Height of rim above ground 0.62 foot, above mean sea level $117 \cdot 7$ feet.

The Campbell-Stokes sunshine recorder is placed on the roof of the Laboratory, at the point where the eye observations of wind, cloud, and weather are made. The instrument fits into a frame mounted on an adjustable stage, so that it can be removed and replaced without further adjustment. The place of observation is in lat. $50^{\circ} 21^{\prime} 49^{\prime \prime} \mathrm{N}$., long. $4^{\circ} 8^{\prime} 21^{\prime \prime} \mathrm{W}$., as determined by measurements from the dial on the Breakwater, of which the position is accurately laid down on the chart.

The hours of observation are 9 a.m. and 9 p.m. local time, or $9 \mathrm{~h} .16 .5 \mathrm{~m}$. Greenwich time. It was found impossible to take regular daily observations with greater frequency.

The ordinary routine work was begun on September 1st, and a table showing the means for the month is appended to these notes.

Mr. Merrifield has been kind enough to give me access to his father's meteorological records, and I hope to be able to discuss them fully in a future number of the Journal. In the meantime a few of the more obvious results may be of interest.

The position of the observing station was in lat. $50^{\circ} 22^{\prime} 25^{\prime \prime} \mathrm{N}$., long. $4^{\circ} 7^{\prime} 16 \cdot 5^{\prime \prime} \mathrm{W}$. From the commencement of the observations the instruments were 90 feet above mean sea level up to July, 1873 . They were then removed to a new position, cistern of barometer 69 feet above mean sea level; rain gauge 9 feet 2 inches above the ground. The hour of observation was $8 \mathrm{a} . \mathrm{m}$. till 1887, when it was changed to 9 a.m. 


\section{Atmospheric Pressure.}

The mean pressures, reduced to $32^{\circ} \mathrm{F}$. and sea level (for the twenty-six years, 1865-1890), are as follows :

\begin{tabular}{|c|c|c|c|c|c|c|c|}
\hline \multirow{2}{*}{ January } & \multirow[b]{2}{*}{. } & \multirow[b]{2}{*}{. } & \multicolumn{2}{|c|}{$\begin{array}{l}\text { Mean pressure, } \\
\text { 8 a.m. }\end{array}$} & & \multicolumn{2}{|c|}{$\begin{array}{l}\text { Monthly } \\
\text { range. }\end{array}$} \\
\hline & & & . $29 \cdot 956$ & nches & $\ldots$ & $1 \cdot 455$ & inch. \\
\hline February & . & . & . $29 \cdot 980$ & " & $\ldots$ & $1 \cdot 277$ & " \\
\hline March & . & . & . $29 \cdot 941$ & " & $\cdots$ & $1 \cdot 258$ & ", \\
\hline April . & . & . & . $29 \cdot 898$ & " & $\ldots$ & $1 \cdot 094$ & , \\
\hline May . & . & . & . $29 \cdot 968$ & " & $\cdots$ & 0.898 & " \\
\hline June . & . & . & . 30.024 & , & $\ldots$ & $0 \cdot 778$ & " \\
\hline July . & . & . & . $29 \cdot 979$ & , & $\cdots$ & $0 \cdot 762$ & " \\
\hline August & . & . & . $29 \cdot 965$ & " & $\ldots$ & 0.761 & " \\
\hline September & . & . & . $29 \cdot 975$ & " & $\cdots$ & 0.927 & ": \\
\hline October & . & . & . $29 \cdot 924$ & " & $\ldots$ & $1 \cdot 171$ & , \\
\hline November & . & - & . $29 \cdot 925$ & " & $\ldots$ & $1 \cdot 257$ & $"$ \\
\hline December & . & - & . $29 \cdot 952$ & $"$ & $\ldots$ & $1 \cdot 277$ & ", \\
\hline & Leans . & . & . $29 \cdot 957$ & " & & 1.076 & " \\
\hline
\end{tabular}

The maximum pressure thus occurs in June, and the minimum in October and November. The month of greatest range is January ; that of least, August. The highest recorded reading, 30.952 inches, occurs in January, 1882 ; and the lowest, $28 \cdot 418$ inches, in December, 1876. The highest monthly mean, 30.347 inches, is that for January, 1880 ; and the lowest, $29 \cdot 422$ inches that for December, 1876 . It should be noted that for the reductions to sea level the tables supplied by the Meteorological Office have been employed.

For purposes of comparison I have taken the means for the fifteen years, 1870-1884, and beside these are placed means for the same years from Falmouth Observatory, the last named being extracted from the "Challenger" Report on Atmospheric Circulation,* and reduced to sea level by means of the table given in 'Instructions in the Use of Meteorological Instruments,' issued by the Meteorological Office.

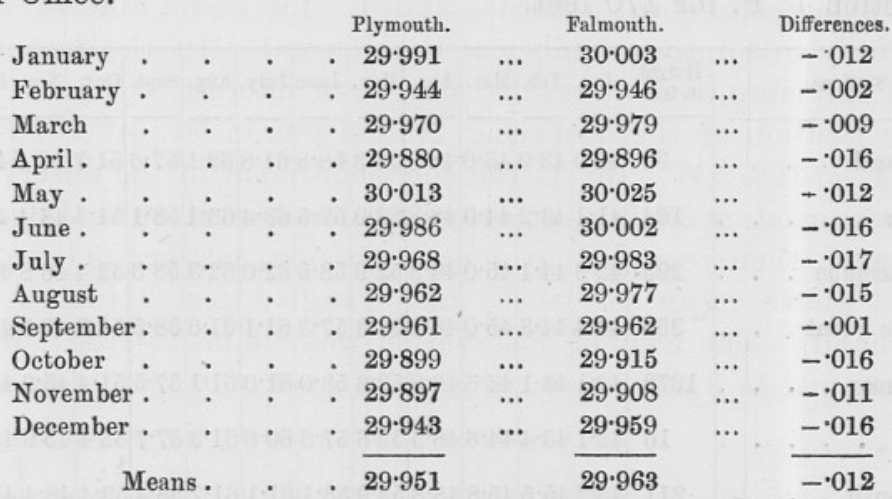

* Voyage of H.M.S. "Challenger," Physics and Chemistry, ii, pt. 5. 


\section{NOTES ON METEOROLOGICAL OBSERTATIONS AT PLYMOUTH.}

The correction for daily range in the above means for Plymouth is small, that derived from the Falmouth observations amounting to +0.003 inch during the month of June. I hope to determine its amount accurately by a full discussion of Dr. Merrifield's observations, supplemented by the readings of a Richard barograph now recording in the Laboratory.

\section{Temperature.}

The mean temperatures given below are calculated from the readings of maximum and minimum self-registering thermometers by the formula T. = Min. + K (Max. - Min.), where $\mathrm{K}$ has the following values :

K.

$\left.\begin{array}{l}\text { January } \\ \text { December }\end{array}\right\} 0.520$
$\left.\begin{array}{l}\text { February } \\ \text { November }\end{array}\right\} 0.500$
$\left.\begin{array}{l}\text { March } \\ \text { October }\end{array}\right\} 0.485$

K.

$\left.\begin{array}{l}\text { April } \\ \text { September }\end{array}\right\} 0 \cdot 476$
$\left.\begin{array}{l}\text { May } \\ \text { August }\end{array}\right\} 0 \cdot 470$
$\left.\begin{array}{l}\text { June } \\ \text { July }\end{array}\right\} 0 \cdot 465$

(See title-page of Weekly Weather Report, 1884, Meteorological Office.)

$$
\text { Mean for Twenty-six Years, 1865-1890. }
$$

\begin{tabular}{|c|c|c|c|c|}
\hline January & . $41 \cdot 9$ & June. & . $59 \cdot 7$ & November. \\
\hline February & . $43 \cdot 1$ & July & . $62 \cdot 6$ & December . \\
\hline arch & . $43 \cdot 9$ & August & . $62 \cdot 0$ & \\
\hline pril & . $49 \cdot 2$ & September. & . 58.2 & $M$ \\
\hline & . 53.8 & October & . $51 \cdot 5$ & \\
\hline
\end{tabular}

Taking the fifteen years 1870-1884 for Plymouth, we may compare with the same period for Exeter, Babbacombe, Prawle Point, Dartmoor (Princetown?), and Bude as give in "Challenger" Report before quoted, reducing each to sea level by means of the correction $1^{\circ} \mathrm{F}$. for 270 feet.

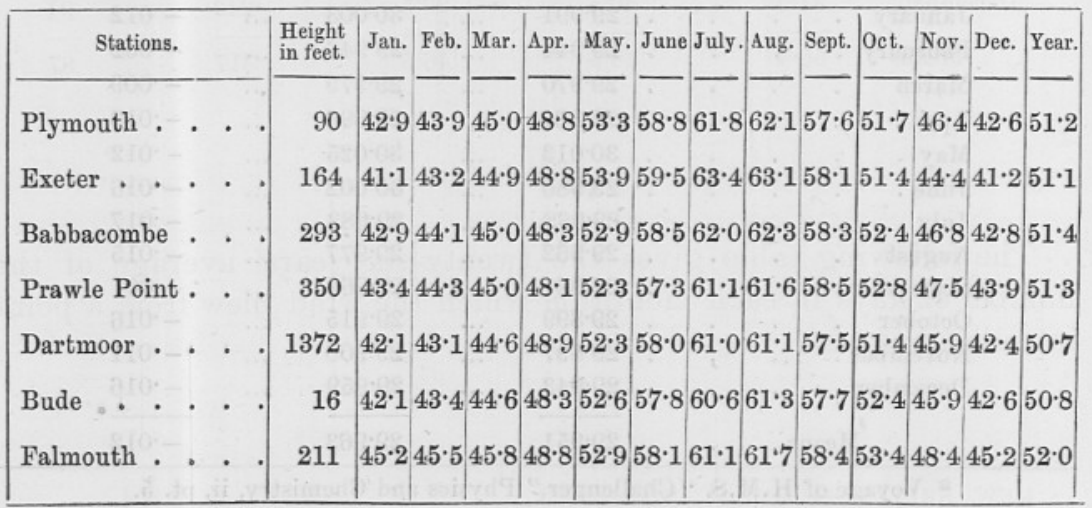


From this table it appears that the curve of temperature at Plymouth is of a form intermediate between stations wholly exposed to the influence of the sea, and stations more inland. Thus on an average Plymouth is about $1^{\circ} \mathrm{F}$. colder than Prawle Point during the winter months, and during the summer months about $1^{\circ} \mathrm{F}$. warmer. On the other hand, Plymouth is $1.5^{\circ} \mathrm{F}$. warmer than Exeter in winter, and in summer $1.5^{\circ}$ colder. In spring and autumn the temperatures of these stations are almost identical, although it may be observed that in September the temperature of Plymouth shows a somewhat greater fall than occurs, e.g., at Babbacombe, a a phenomenon which, although not fully confirmed by the mean of the longer period, suggests the influence of the high plateau of Dartmoor in some peculiar seasons.

\section{Humidity.}

The hygrometric conditions are shown by the following readings of dry and wet bulb thermometers, the values being means for twenty-six years as before.

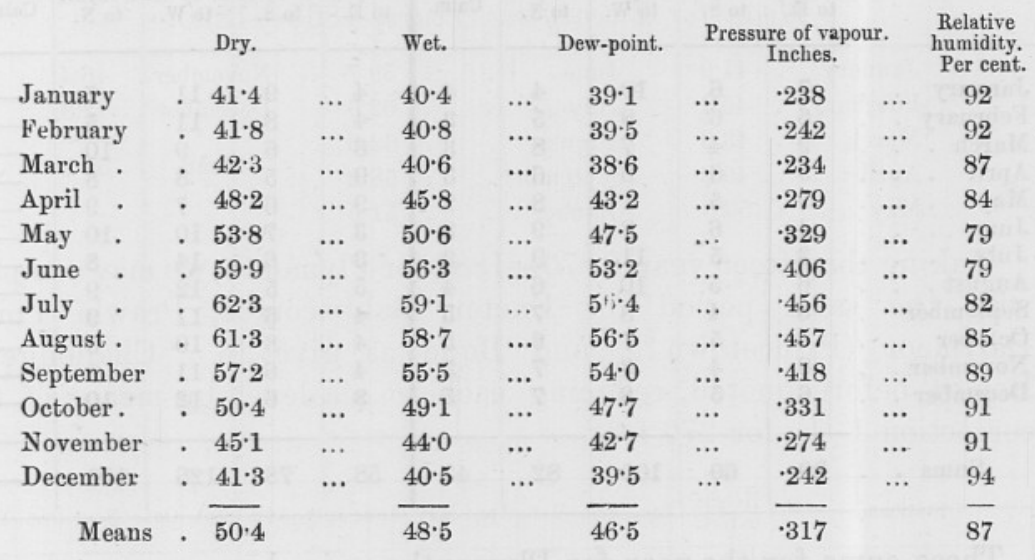

\section{Winds.}

The following table gives the twenty-six years' average of the number of days in each month on which the wind blew from a point in each quadrant. 
NOTES ON METEOROLOGICAL OBSERVATTONS AT PLYMOUTH.

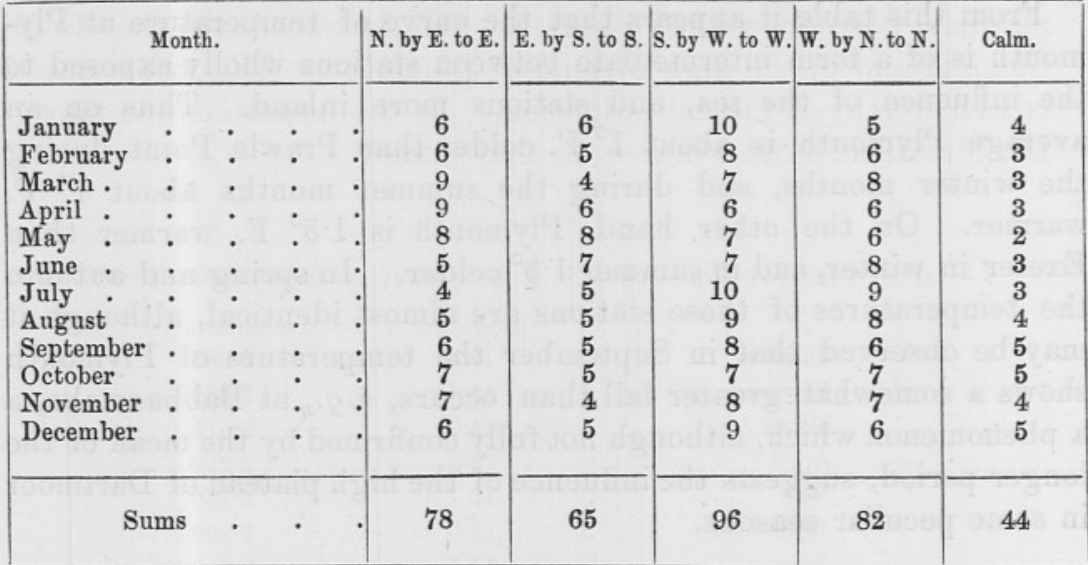

Taking the fifteen years $1870-1884$ as before, and summing the values N.E. and E., S.E. and S., S.W. and W., N.W. and N., given in the "Challenger" Reports, for Falmouth at 8 a.m., we get the following :

\begin{tabular}{|c|c|c|c|c|c|c|c|c|c|c|c|}
\hline \multirow[b]{2}{*}{ Month. } & & \multicolumn{5}{|c|}{ Plymouth. } & \multicolumn{5}{|c|}{ Falmouth. } \\
\hline & & $\begin{array}{c}\text { N. by } E \text {. } \\
\text { to E. }\end{array}$ & $\begin{array}{l}\text { E. by } S . \\
\text { to } \mathrm{S} \text {. }\end{array}$ & $\begin{array}{l}\text { S. by W. } \\
\text { to W. }\end{array}$ & $\begin{array}{c}\text { W. by N. } \\
\text { to N. }\end{array}$ & Calm. & $\begin{array}{c}\text { N. by E. } \\
\text { to E. }\end{array}$ & $\begin{array}{l}\text { F. by S. } \\
\text { to } \mathrm{S} \text {. }\end{array}$ & $\begin{array}{l}\text { s. by W. } \\
\text { to W. }\end{array}$ & $\begin{array}{l}\text { W. by N. } \\
\text { to N. }\end{array}$ & Calm. \\
\hline January & & 7 & 6 & 10 & 4 & 4 & 4 & 9 & 11 & 7 & - \\
\hline February & & 6 & 6 & 8 & 5 & 3 & 4 & 8 & 11 & 5 & - \\
\hline March. & 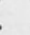 & 9 & 4 & 7 & 8 & 3 & 6 & 6 & 9 & 10 & - \\
\hline April . & • & 9 & 6 & 6 & 6 & 3 & 9 & 5 & 8 & 8 & - \\
\hline May. . & & 9 & 5 & 7 & 8 & 2 & 9 & 6 & 7 & 9 & - \\
\hline June . & & 4 & 6 & 8 & 9 & 3 & 3 & 7 & 10 & 10 & - \\
\hline July . & & 3 & 5 & 11 & 9 & 3 & 3 & 6 & 14 & 8 & - \\
\hline August. & . & 6 & 5 & 10 & 6 & 4 & 5 & 5 & 12 & 9 & - \\
\hline September & & 6 & 4 & 8 & 7 & 5 & 4 & 6 & 11 & 9 & - \\
\hline October & . & 8 & 5 & 7 & 6 & 5 & 4 & 8 & 10 & 9 & - \\
\hline November & • & 8 & 4 & 8 & 7 & 3 & 4 & 6 & 11 & 9 & - \\
\hline December & . & 6 & 5 & 8 & 7 & 5 & 3 & 6 & 12 & 10 & - \\
\hline Sums & • & 80 & 60 & 100 & 82 & 43 & 58 & 78 & 126 & 103 & - \\
\hline
\end{tabular}

These sums for the year for Plymouth are in close agreement with those for the longer period, and we observe, as compared with Falmouth, a considerable average of calms, and excess of northeasterly winds, which again point to the influence of high land as hinted in the temperature curve.

\section{Rainfall.}

The rainfall of Plymouth is an element the discussion of which raises many problems of the greatest interest, on account of its 
NOTES ON METEOROLOGIOAL OBSERVATIONS AT PLYMOUTH. 177

peculiar position with respect to the entrance to the English Channel and to the high plateau of Dartmoor. The whole question is well worthy of full investigation. In the meantime one may give Dr. Merrifield's observations as they stand.

Averages for Twenty-six Years ending December, 1890.

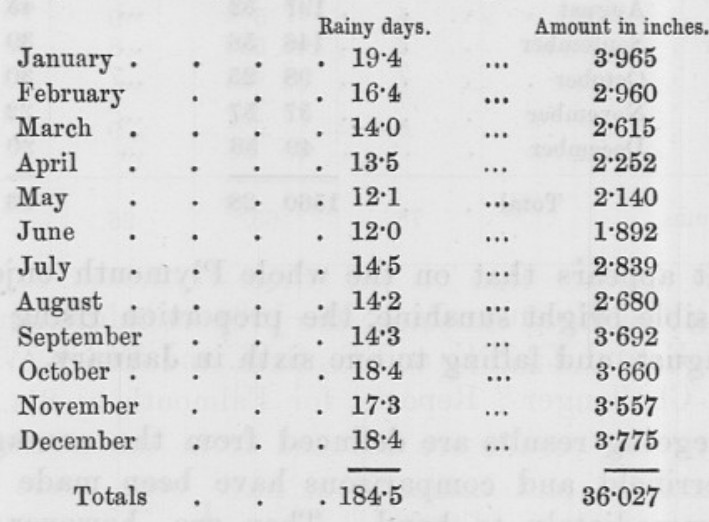

The following notes are given by Dr. Merrifield :- "The average for five years is taken from the Quarterly Weather Report, Meteorological Office, January to March, 1870 ; rain gauge not used until January, 1869. In ten years from 1869 to 1878, Mr. Balkwill, Old Town Street (Plymouth), had 412.05 inches; Navigation School, 363.19 inches. Hence 13.45 per cent. should be added to Navigation School amounts. In five years from 1870 to $1874 \mathrm{Mr}$. Balkwill had 196.94 inches; Navigation School, $171 \cdot 09$, or add $15 \cdot 11$ per cent. to Navigation School; Drake's reservoir, 201.43 inches and 895 rainy days; Navigation School, 171.09 inches and 865 rainy days. Hence add 17.73 per cent. rain and 3.47 per cent. number of rainy days to Navigation School."

\section{Sunshine.}

Dr. Merrifield's records of the duration of sunshine begin with the year 1882 . We have accordingly nine complete years, with the following results : 
178 NOTES ON METEOROLOGICAL OBSERVATIONS AT PLYMOUTH.

\begin{tabular}{|c|c|c|c|c|c|}
\hline & & $\begin{array}{c}\text { Total su } \\
\text { Hrs. }\end{array}$ & $\begin{array}{l}\text { inshine. } \\
\text { Min. }\end{array}$ & & $\begin{array}{l}\text { Percentage } \\
\text { of possible. }\end{array}$ \\
\hline January . & . & . 41 & 33 & $\ldots$ & 16 \\
\hline February & . & . 76 & 48 & $\ldots$ & 27 \\
\hline March . & . & .130 & 27 & $\ldots$ & 36 \\
\hline April & . & .171 & 53 & $\ldots$ & 42 \\
\hline May & . & . 198 & 25 & $\ldots$ & 40 \\
\hline June & . & . 202 & 59 & $\cdots$ & 42 \\
\hline July & . & .187 & 17 & $\ldots$ & 38 \\
\hline August . & . & . 197 & 52 & $\ldots$ & 45 \\
\hline September & . & . 146 & 56 & $\ldots$ & 39 \\
\hline October . & . & . 98 & 25 & $\cdots$ & 30 \\
\hline November & . & . 57 & 57 & $\ldots$ & 22 \\
\hline December & - & . 49 & 56 & $\cdots$ & 20 \\
\hline Tota & ${ }^{\circ}$ & 1560 & 28 & - & 33 \\
\hline
\end{tabular}

Whence it appears that on the whole Plymouth enjoys one third of the possible bright sunshine, the proportion rising to nearly one half in August, and falling to one sixth in January.

The foregoing results are deduced from the averages computed by Dr. Merrifield, and comparisons have been made only with the material immediately to hand. They are, however, sufficient to show the great value of Dr. Merrifield's records, and of what may be expected from a full discussion. 
Meteorological Observations at M.B.A. Laboratory, Plymouth, September, 1891, 9 a.m. and 9 p.m.

\begin{tabular}{|c|c|c|c|c|c|c|c|c|c|c|c|c|c|c|c|c|c|}
\hline & & & & $\left\{\begin{array}{l}\text { Lat. } \\
\text { Lon }\end{array}\right.$ & t. 50 & $\begin{array}{ll}0^{\circ} \\
4 . \\
4 . & 21^{\prime} \\
8^{\prime}\end{array}$ & $\begin{array}{l}49^{\prime \prime} \mathrm{N} \\
21^{\prime \prime} \mathrm{V}\end{array}$ & $\left.\begin{array}{l}\text { N. } \\
\text { W. }\end{array}\right\}$ & & \multicolumn{8}{|c|}{$\begin{array}{l}\text { Height of cistern of barometer above mean sea level } 125.93 \text { feet. } \\
, \quad \text { rain gauge }\end{array}$} \\
\hline \multirow{3}{*}{ 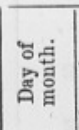 } & \multirow{3}{*}{ 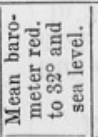 } & \multicolumn{5}{|c|}{ Temperature. } & \multirow{3}{*}{ 䖯 } & \multirow{3}{*}{ 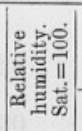 } & \multicolumn{3}{|c|}{ Wind. } & \multirow{2}{*}{\multicolumn{2}{|c|}{$\mid \begin{array}{c}\text { Cloud } \\
\text { amount, } \\
0-10 .\end{array}$}} & \multirow{3}{*}{ 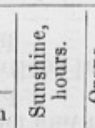 } & \multirow{3}{*}{ हैं } & & \multirow{3}{*}{ Remarks. } \\
\hline & & & & & & & & & \multicolumn{2}{|c|}{ Direction. } & \multirow{2}{*}{\begin{tabular}{|c|}
$\begin{array}{c}\text { Mean } \\
\text { force, } \\
0-12\end{array}$ \\
$0-12$
\end{tabular}} & & & & & & \\
\hline & & $\begin{array}{l}\text { Dry } \\
\text { bulb. }\end{array}$ & $\begin{array}{l}\text { wet } \\
\text { bulb. }\end{array}$ & Max. & Min. & $\begin{array}{l}\text { Dew- } \\
\text { point. }\end{array}$ & & & 9 a.m. & 9 p.m. & & 9 a.m. & 9 p.m & & & & \\
\hline 1 & $29 \cdot 627$ & $57 \cdot 7$ & $55 \cdot 3$ & $62 \cdot 4$ & $56 \cdot 0$ & $053 \cdot 1$ & $\cdot 405$ & \begin{tabular}{|c|c|}
5 & 84
\end{tabular} & S.S.W. & S.S.W. & 2 & 10 & 0 & - & 4 & .000 & Gale in forenoon. \\
\hline 2 & 29.872 & $56 \cdot 2$ & $55 \cdot 0$ & $60 \cdot 2$ & 54.7 & $754 \cdot 0$ & $\mid 418$ & \begin{tabular}{l|l|}
8 & 92 \\
3
\end{tabular} & S.S.W. & Calm & 1 & 10 & 0 & $1 \cdot 70$ & 1 & .000 & roreno \\
\hline 3 & $29 \cdot 979$ & 57.7 & $55 \cdot 2$ & $61 \cdot 6$ & 53.8 & 852.9 & $\cdot 402$ & 83 & S.E. & S.W. & 1 & 2 & 1 & $10 \cdot 27$ & 6 & .000 & aight. \\
\hline 4 & $30 \cdot 102$ & $56 \cdot 7$ & $754 \cdot 8$ & & 54.7 & 753.1 & $\cdot 405$ & 84 & s.W. & S.W. & 2 & 7 & 0 & $9 \cdot 34$ & 4 & .000 & \\
\hline 5 & $30 \cdot 198$ & $57 \cdot 1$ & $53 \cdot 3$ & $61 \cdot 2$ & 53.8 & $\begin{array}{lll}8 & 49 \cdot 9\end{array}$ & $\cdot 360$ & 77 & s.w. & S.W. & 3 & 7 & 0 & $4 \cdot 61$ & 5 & .000 & \\
\hline 6 & $30 \cdot 015$ & $57 \cdot 3$ & $36 \cdot 2$ & $61 \cdot 5$ & $55 \cdot 6$ & $655 \cdot 2$ & $\cdot 432$ & 93 & S.W. $\times$ W. & S.W. $\times$ S & 4 & 10 & 10 & $2 \cdot 73$ & 6 & $\cdot 275$ & \\
\hline 7 & $30 \cdot 027$ & $58 \cdot 2$ & $58 \cdot 1$ & $62 \cdot 1$ & $56 \cdot 4$ & $458 \cdot 0$ & $\cdot 482$ & 100 & S.S.W. & S.S.W. & 1 & 10 & 10 & $0 \cdot 71$ & 7 & .025 & \\
\hline 8 & & $60 \cdot 6$ & $58 \cdot 1$ & $64: 0$ & $57 \cdot 1$ & 155.9 & $\cdot 449$ & 85 & s. & N.E. & 2 & 0 & 9 & $5 \cdot 10$ & 6 & .010 & \\
\hline 9 & $\begin{array}{l}30 \cdot 092 \\
\end{array}$ & $64 \cdot 6$ & $600 \cdot 7$ & $73 \cdot 4$ & 558 & 857.5 & .474 & $\begin{array}{r}79 \\
+\end{array}$ & E.S.E. & N.E. $\times$ N. & 1 & 1 & 0 & 11.05 & $\begin{array}{l}0 \\
4 .\end{array}$ & .000 & \\
\hline 10 & $\begin{array}{l}30.093 \\
0.03\end{array}$ & 35.6 & $659 \cdot 9$ & 73.6 & 59.8 & $\begin{array}{ccc}8 & 55 \cdot 4\end{array}$ & 439 & 70 & E.N.E. & Calm & 1 & 0 & 0 & 11.51 & $\frac{4}{2}$ & .000 & \\
\hline 11 & 30.097 & $66 \cdot 6$ & $660 \cdot 3$ & $75 \cdot 2$ & 53.9 & $955 \cdot 4$ & 440 & 68 & E.N.E. & E. $\times$ N. & 1 & 0 & 0 & $11 \cdot 10$ & 1 & .000 & \\
\hline 12 & $30 \cdot 004$ & $69 \cdot 0$ & $62 \cdot 0$ & & & & $\cdot 459$ & 65 & E. $\times N$. & N.E. & 1 & 0 & 0 & $11 \cdot 17$ & & & Faint lunar halo p.m. \\
\hline 13 & $29 \cdot 812$ & $63 \cdot 5$ & 5009 & $70 \cdot 6$ & 60.9 & 958.6 & 494 & 85 & E.S.E. & E.N.E. & 1 & 7 & 4 & 1.04 & 6 & .004 & \\
\hline 14 & & $57 \cdot 4$ & $455 \cdot 2$ & $62 \cdot 6$ & $53 \cdot 1$ & $153 \cdot 2$ & 414 & 86 & E.S.E. & w. & 1 & 10 & 0 & $2 \cdot 17$ & 7 & .040 & Wind force 7 in afternoon. \\
\hline 15 & & $96 \cdot 0$ & $053 \cdot 4$ & $61 \cdot 3$ & $47 \cdot 8$ & $81 \cdot 0$ & 376 & 84 & $\mathrm{~W}$ & Calm & 1 & 8 & 9 & $3 \cdot 37$ & 1 & .002 & \\
\hline 16 & 3 & $57 \cdot 1$ & 155.6 & $60 \cdot 5$ & $48 \cdot 8$ & $854: 3$ & $\cdot 422$ & $\begin{array}{r}90 \\
2\end{array}-x$ & & W.S.W. & 1 & 10 & 8 & 0.00 & 7 & $.0 \mathrm{co}$ & $\mathrm{Lu}$ \\
\hline 17 & 30 & $59 \cdot 6$ & $658 \cdot 6$ & $62 \cdot 6$ & 552 & 27.7 & $\cdot 478$ & \begin{tabular}{|c|c|c|}
9 & 94
\end{tabular} & W. & S.W. & 4 & 7 & 10 & $2 \cdot 97$ & 5 & $\cdot 147$ & \\
\hline 18 & 30 & & $\begin{array}{l}30.3 \\
60 \cdot 3\end{array}$ & $62 \cdot 3$ & & & .523 & $\begin{array}{r}97 \\
100\end{array}$ & W. & Cal & 1 & 10 & 10 & 0.00 & 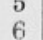 & .470 & \\
\hline 19 & 29 & $59 \cdot 4$ & $459 \cdot 4$ & 61.8 & $58 \cdot 4$ & $459 \cdot 4$ & .507 & 100 & s.W. & S. $x$ & 2 & 10 & 10 & 0.00 & 6 & 270 & \\
\hline 20 & & $55 \cdot 0$ & $052 \cdot 4$ & $59 \cdot 8$ & 52.5 & & 361 & 83 & & W.N.W. & 2 & 9 & 9 & $3 \cdot 12$ & 6 & .194 & 4. Wind force 7 at 2 a.m. Bar. $\min .5$ a.m. \\
\hline 21 & 29 & $51 \cdot 6$ & $650 \cdot 1$ & $58 \cdot 2$ & 48.9 & 948.5 & .342 & 89 & W.S.W. & $\mathrm{N}$ & 2 & 10 & 10 & $4 \cdot 18$ & 4 & .039 & $9 \mathrm{~W}$ \\
\hline 22 & & 54.5 & $51 \cdot 4$ & $61 \cdot 4$ & $50 \cdot 9$ & $948 \cdot 5$ & 343 & 0 & N. & N.1 & 2 & 7 & 0 & $8 \cdot 00$ & 3 & .000 & \\
\hline 23 & 3 & 54.0 & $52 \cdot 5$ & $62 \cdot 1$ & 44.6 & 61.2 & -378 & $\begin{array}{l}30 \\
3\end{array}$ & E.N.E. & N.N.E. & 0 & 0 & 0 & $9 \cdot 12$ & 7 & $\cdot 378$ & ght. \\
\hline 24 & & $57 \cdot 0$ & $057 \cdot 0$ & $59 \cdot 2$ & 50.8 & 87.0 & -466 & 100 & & Caim & 3 & 10 & 10 & 0.08 & 7 & $\cdot 100$ & \\
\hline 25 & $30 \cdot 115$ & 57.9 & 957.4 & $59 \cdot 4$ & 56.9 & 56.9 & $\begin{array}{l}.400 \\
\cdot 465\end{array}$ & 97 & s. & dat & 1 & 10 & $\begin{array}{r}10 \\
0\end{array}$ & 0.02 & 6 & .030 & \\
\hline 26 & $29 \cdot 931$ & $57 \cdot 4$ & 455 & $62 \cdot 2$ & 55.6 & $\begin{array}{l}637 \\
537\end{array}$ & \begin{tabular}{|c|}
.415 \\
.415
\end{tabular} & 88 & S.w. & W. & 3 & 10 & 10 & $1 \cdot 20$ & 4 & .000 & \\
\hline 27 & & 54.9 & $52 \cdot 0$ & & $52 \cdot 0$ & $49 \cdot 3$ & $\begin{array}{l}352 \\
352\end{array}$ & 82 & & W.S.W. & 2 & 8 & 0 & 8.06 & 7 & 00 & \\
\hline 28 & $30 \cdot 156$ & $58 \cdot 1$ & 56.7 & & & $55 \cdot 3$ & $\begin{array}{l}.00 \\
-437 \\
\end{array}$ & 90 & s. & S & 2 & 8 & 0 & 8.50 & 6 & .000 & \\
\hline 29 & & $56 \cdot 6$ & 560 & $59 \cdot 2$ & 55.4 & 455 & 442 & 96 & & S.W. & 3 & 10 & 3 & 0.00 & 8 & $\cdot 102$ & $2 \mathrm{Wi}$ \\
\hline 30 & 29.814 & 57.7 & $755 \cdot 9$ & $61 \cdot 2$ & $47 \cdot 7$ & $754 \cdot 4$ & 424 & 89 & S. $\times W$. & S.S.W. & 5 & 8 & 0 & $8 \cdot 17$ & 4 & $\cdot 447$ & 7 Gale from midnight till 5 a.m. on 1 st. \\
\hline Means & $30 \cdot 027$ & $58 \cdot 5$ & $56 \cdot 3$ & $63 \cdot 4$ & $53 \cdot 7$ & $54 \cdot 4$ & $\cdot 4278$ & $86 \cdot 8$ & & & 1.9 & 7 & 4 & $5 \cdot 01$ & 5 & 084 & \\
\hline
\end{tabular}

Rain on 14 days.

Wind.

Fog on 9 days.

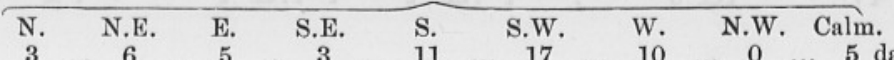


Notes on the Herring, Long-Line, and Pilchard Fisheries of Plymouth (continued).

By Willam Roach,

Associate Member.

I.-Herring

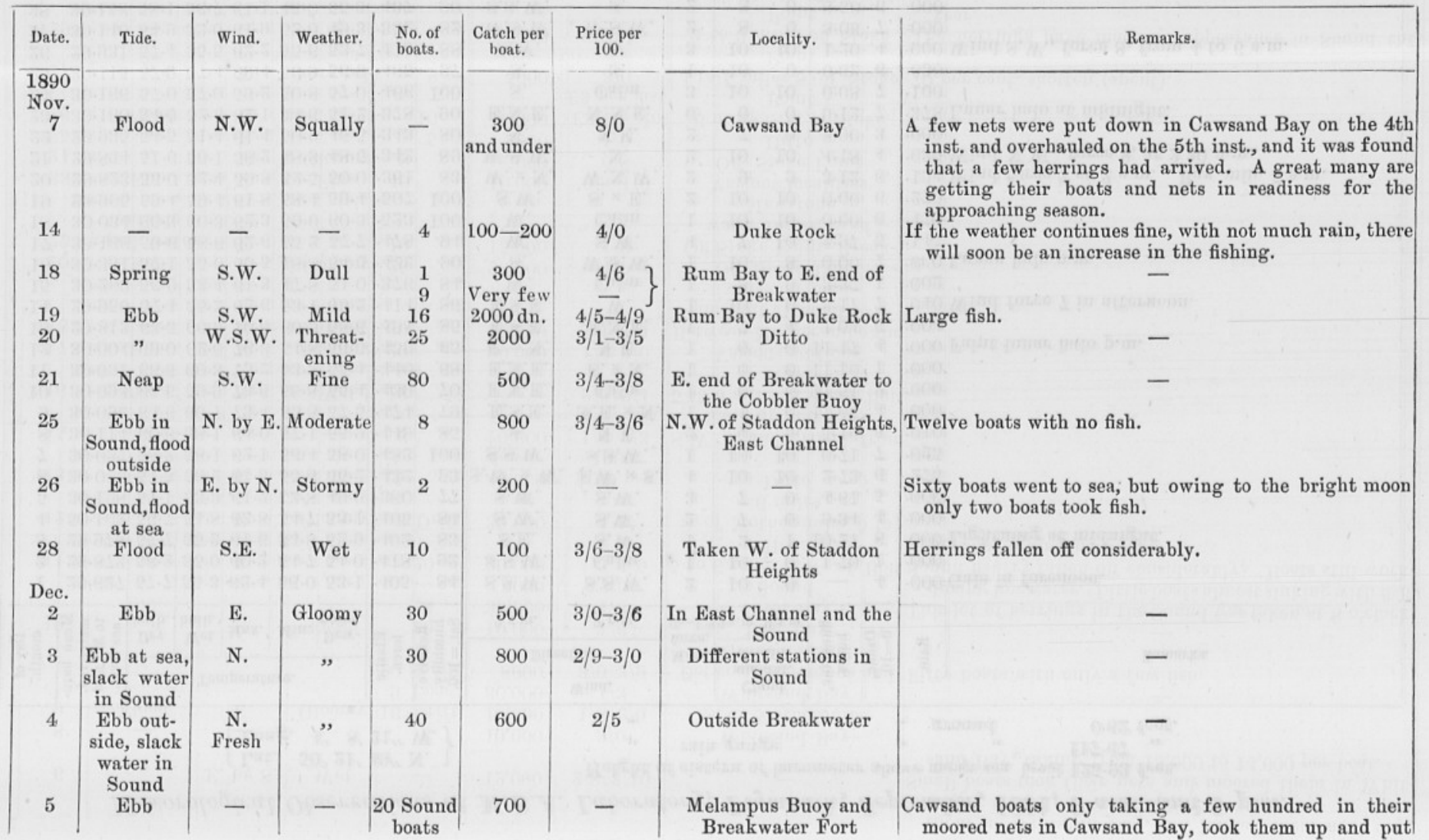


them in Whitsand Bay, and hauled them next day, taking from 10,000 to 12,000 .

Small boats took their nets and moored them in Whit sand Bay, taking from 13,000 to 14,000 per boat.

Whitsand Bay

S. of Mothecombe

Whitsand Bay

Between Melampus and Fifty boats with only a few fish.

Drake's Island

East Bay

West Bay

Sound

This lot of herrings in the Sound was taken at 8 o'clock,

during low water. Little boats almost sinking with fish.
Sound fishery fallen off considerably. Boats still work-

$8000 \quad 2 / 0$

20,000

$1 / 3-1 / 9$

to 6 miles S.W. S. of Mothecom

ing, but taking no fish.

E. Gloomy 3 Drift

40,000

$10 / 0$ per

(1)

Ditto

\begin{tabular}{c|c|cc|cc} 
E. & Fine & 15 &, & $\begin{array}{l}40,000 \\
\text { down }\end{array}$ & $10 / 0$ per \\
& & & & 1000 \\
- & - & 6 &, & 10,000 & $1 / 6-2 / 3$
\end{tabular}

-

S.W. of Bolt Head, from
there up and down

2/0-2/4 5 to 6 miles S.W. of Bolt

$$
\begin{aligned}
& \text { iles S.W. } \\
& \text { Head }
\end{aligned}
$$

$20,000 \quad 1 / 6-3 / 0$

Various stations

Between Mothecombe and

the Bolt Head, 4 to 5

miles off land

Off Stoke Point, Break-

water Light, S. of

Mewstone

Various localities

Off Stoke Point

Ditto

$5000 \quad 0 / 8$

$10,000 £ 210$ s. to

Flood

E. Gloomy 8 ,"

E. ",

E. Very cold

$£ 3$ per last

6 miles S.E. of Stoke

4 to 5 Point

$5000 \quad 3 / 0$

to 5 miles S. of Stoke 70 per cent. shotten (spent).

Point

$3000 \quad 2 / 10$

E. Very cold

50

Ebb

20,000

2 to 3 miles off Stoke

The herrings have made no appearance in Sound this Point year.

$30,000 \quad 2 / 8-2 / 10$

2 to 3 miles $S$. of Salcombe

Ditto

Two boats lost half their fleet of nets on account of
heavy takes. 


\begin{tabular}{|c|c|c|c|c|c|c|c|c|}
\hline Date. & Tide. & Wind. & Weather. & $\begin{array}{l}\text { No. of } \\
\text { boats. }\end{array}$ & $\begin{array}{c}\text { Catch per } \\
\text { boat. }\end{array}$ & $\begin{array}{l}\text { Price per } \\
100 .\end{array}$ & Locality. & Remarks. \\
\hline 1891 & & & & & & . & & \\
\hline$\underset{1}{\text { Jan. }}$ & Ebb & E. & $\begin{array}{l}\text { Threat- } \\
\text { ening }\end{array}$ & 5 & 12,000 & $\begin{array}{l}\text { Full } 3 / 0, \\
\text { shotten } \\
1 / 1-2 / 0\end{array}$ & Off Mothecombe & 60 per cent. shotten. \\
\hline 2 & , & E. & Fine & 30 & 20,000 & $2 / 6-3 / 6$ & $\begin{array}{l}\text { Off Mothecombe, Break- } \\
\text { water Light, between the } \\
\text { Mewstone and land }\end{array}$ & - \\
\hline 3 & , & E. & Gloomy & 10 & 10,000 & $\begin{array}{c}\text { Full } 3 / 1 \\
\text { shotten } 2 / 0\end{array}$ & Same as 2nd inst. & - \\
\hline 6 & $\begin{array}{c}\text { Slack water } \\
\text { outside, } \\
3 \text { hours ebb }\end{array}$ & E. & Moderate & 40 & 40,000 & $\begin{array}{c}\text { Full } \\
3 / 0-3 / 6 \\
\text { shotten }\end{array}$ & $\begin{array}{c}\text { Between Stoke Point and } \\
\text { Bolt Head, } 4 \text { to } 8 \text { miles } \\
\text { off land }\end{array}$ & - \\
\hline 7 & $\begin{array}{l}\text { in Sound } \\
\text { 1st hour } \\
\text { flood }\end{array}$ & N.E. & Cold & 30 & 25,000 & $\begin{array}{l}1 / 6-2 / 0 \\
1 / 8-2 / 3\end{array}$ & Ditto & 70 to 80 per cent. shotten. \\
\hline 8 & $\begin{array}{l}\text { 2nd hour } \\
\text { flood }\end{array}$ & s. & $\begin{array}{l}\text { Threat- } \\
\text { ening }\end{array}$ & 20 & 15,000 & $\begin{array}{c}\text { Full } 2 / 0, \\
\text { shotten } 1 / 6\end{array}$ & $\begin{array}{c}\text { From Whitsand Bay to } \\
\text { Bolt Head }\end{array}$ & One or two shoals travelling west. \\
\hline 10 & Flood & N.E. & Fine & 20 & 20,000 & $1 / 8-2 / 3$ & $\begin{array}{c}\text { Between Stoke Point and } \\
\text { Bolt Head, } 1 \text { to } 2 \text { miles } \\
\text { off land }\end{array}$ & - \\
\hline 12 & , & N. & Gloomy & 10 & $20,000 \mathrm{dn}$. & $1 / 6-2 / 4$ & Ditto & - \\
\hline 13 & $"$ & N. & , & 30 & 15,000 & $1 / 4-2 / 2$ & Same as 12 th inst. & G - \\
\hline 14 & " & N. & Bright & 10 & 20,000 & $1 / 5-1 / 11$ & $\begin{array}{l}1 \frac{1}{2} \text { miles E. of Stoke } \\
\text { Point }\end{array}$ & $\begin{array}{l}\text { It is many years ago since such numbers of herrings } \\
\text { have appeared in the East Bay. }\end{array}$ \\
\hline 15 & Slack water & E. & , & 30 & 30,000 & $1 / 5-2 / 0$ & $\begin{array}{c}1 \text { to } 3 \text { miles S. of Stoke } \\
\text { Point, from there to } \\
\text { Bolt Head }\end{array}$ & \\
\hline 16 & Ebb & E. & , & 15 & 10,000 & $1 / 8-2 / 0$ & $\begin{array}{l}1 \text { to } 3 \text { miles S. of Stoke } \\
\text { Point }\end{array}$ & - \\
\hline 17 & ,, & E. & & 10 & 10,000 & $2 / 0-3 / 0$ & Ditto & - \\
\hline 20 & , & S.W. & Stormy & 6 & 1000 & $2 / 0$ & $\begin{array}{l}8 \text { to } 9 \text { miles outside the } \\
\text { Eddystone }\end{array}$ & - \\
\hline 26 & - & - & - & 20 & 400 & $1 / 6$ & In East Bay & - \\
\hline
\end{tabular}




$$
\text { II.-Long-Line. }
$$

The "Remarks" show very clearly how much difficulty is experienced in procuring suitable bait, and how this branch of the fishing industry is paralysed for want of it. The conclusion might also be drawn that salted pilchard makes but an indifferent bait.

\begin{tabular}{|c|c|c|c|c|c|c|c|c|c|c|c|c|c|}
\hline Date. & Tide. & Wind. & Weather. & Bait. & Locality. & $\begin{array}{l}\text { No. of } \\
\text { boats. }\end{array}$ & Conger. & Ling. & $\begin{array}{l}\text { Rays and } \\
\text { skates. }\end{array}$ & Cod. & Pollack. & $\begin{array}{l}\text { Other } \\
\text { fish. }\end{array}$ & Remarks. \\
\hline 1890 & & & & & & & & & & & & & \\
\hline $\begin{array}{l}\text { Oct. } \\
21\end{array}$ & Ebb & N.W. & Fine & Squid & $\begin{array}{l}\frac{3}{4} \text { mile W. of the } \\
\text { Eddystone }\end{array}$ & 1 & $\begin{array}{l}\text { cwt. } \\
3\end{array}$ & 12 & $\begin{array}{c}\text { Dozen. } \\
3\end{array}$ & - & 1 doz. & $\begin{array}{l}3 \text { doz. } \\
\text { bream }\end{array}$ & - \\
\hline 22 & ”, & E. & , & , & $\begin{array}{l}6 \text { miles S.E. of the } \\
\text { Mewstone }\end{array}$ & 1 & , & 6 & 3 & 2 & , & - & - \\
\hline , & - & 一 & - & Pilchard & 6 miles outside the & e 1 & - & $700-80$ & 0 spur 0 & $\log$-fish & - & - & Nothing but spur dogs (Acanthias) \\
\hline 29 & Flood & W. & Wet & Squid & $\begin{array}{l}1 \text { mile N.W. of the } \\
\text { Eddystone }\end{array}$ & e 1 & 5 & 12 & $1 \mathrm{doz}$. & 2 & $2 \mathrm{doz}$. & $\begin{array}{l}2 \text { doz. } \\
\text { bream }\end{array}$ & - \\
\hline 31 & , & W. & Squally & , & $\begin{array}{l}3 \text { to } 4 \text { miles S.W. } \\
\text { of the Bolt Head }\end{array}$ & 3 & 5 & 5 to 6 & 1 to 2 & 2 to 3 & $\begin{array}{l}1 \text { to } 2 \\
\text { doz. }\end{array}$ & $\begin{array}{l}1 \mathrm{doz} . \\
\text { bream }\end{array}$ & $\begin{array}{l}\text { Weather extremely bad for long-line } \\
\text { fishing. }\end{array}$ \\
\hline $\begin{array}{c}\text { Nov. } \\
5\end{array}$ & - & - & - & Squid & $\begin{array}{l}3 \text { to } 1 \frac{1}{2} \text { miles S.E. } \\
\text { of the Eddystone }\end{array}$ & 3 & 4 & $\begin{array}{l}10 \text { to } \\
12\end{array}$ & 2 to 3 & 3 to 4 & $1 \mathrm{doz}$. & - & - \\
\hline 14 & Flood & s. & Wet & - & - & - & - & - & - & 一 & - & - & - \\
\hline 17 & ," & $\begin{array}{c}\text { S., } \\
\text { moderate }\end{array}$ & Dull & Squid & $\begin{array}{l}5 \text { to } 6 \text { miles S.E. } \\
\text { of Stoke Point }\end{array}$ & 3 & 3 & 7 to 8 & 2 to 4 & 3 to 4 & 5 to 6 & - & - \\
\hline 19 & Ebb & S.W. & Mild & - & On East Reites & 1 & 2 & 12 & 2 & - & - & - & - \\
\hline 21 & - & - & - & - & $\begin{array}{l}\text { Outside the } \\
\text { Eddystone }\end{array}$ & 2 & - & - & 3 to 4 & - & - & - & $\begin{array}{l}\text { Caught } 100 \text { spur dog-fish. There are } \\
\text { a great number on the coast just } \\
\text { now. }\end{array}$ \\
\hline 28 & Flood & N.E. & $\begin{array}{l}\text { Very } \\
\text { cold }\end{array}$ & - & - & 3 & 3 & 6 & 1 to 2 & 6 & - & - & $\begin{array}{l}\text { These boats only shot half their gear } \\
\text { owing to strong wind. }\end{array}$ \\
\hline $\begin{array}{c}\text { Dec. } \\
1\end{array}$ & - & - & - & Squid & $\begin{array}{c}7 \text { to } 8 \text { miles S. of } \\
\text { Bolt Head }\end{array}$ & 2 & $\begin{array}{l}14 \text { to } \\
15\end{array}$ & - & - & - & - & - & 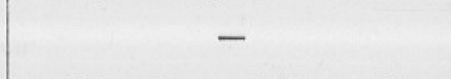 \\
\hline & & & & & & $\begin{array}{l}1 \\
1\end{array}$ & $\begin{array}{l}5 \\
4\end{array}$ & 6 & 2 to 3 & 6 & - & - & $\begin{array}{l}4 \text { to } 5 \text { hand-liners, which use } 4 \text { lines, } \\
\text { landed } 4 \text { cwt. each. }\end{array}$ \\
\hline 3 & Ebb & N. & Gloomy & , & $\begin{array}{l}7 \text { to } 8 \text { miles off } \\
\text { the Bolt Head }\end{array}$ & 4 & 8 & 12 & 2 to 4 & 12 & - & - & - \\
\hline 4 & , & N. & ", & ," & , & 2 & 4 to 6 & 2 & 2 to 4 & 12 & - & - & - \\
\hline
\end{tabular}




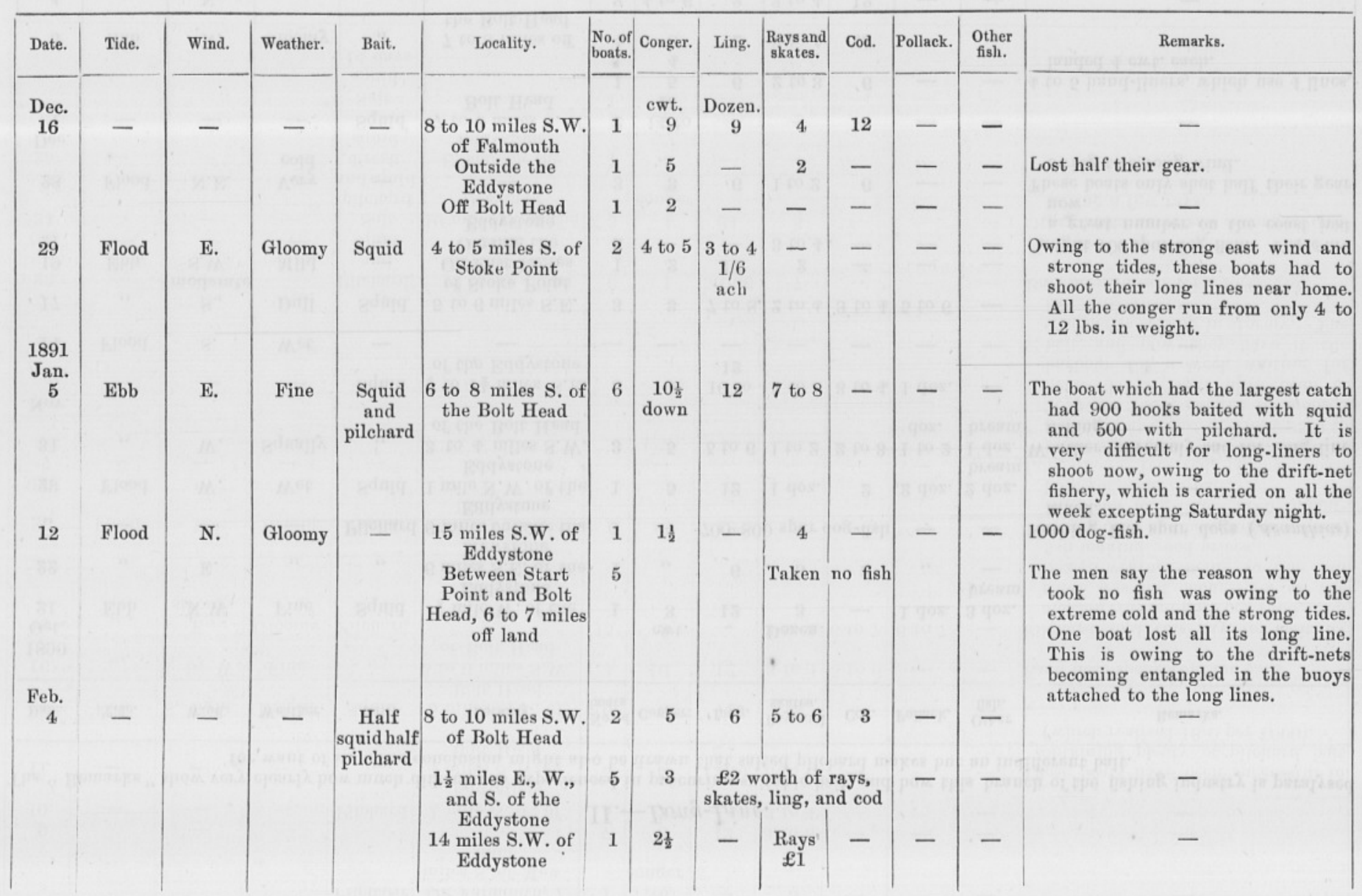




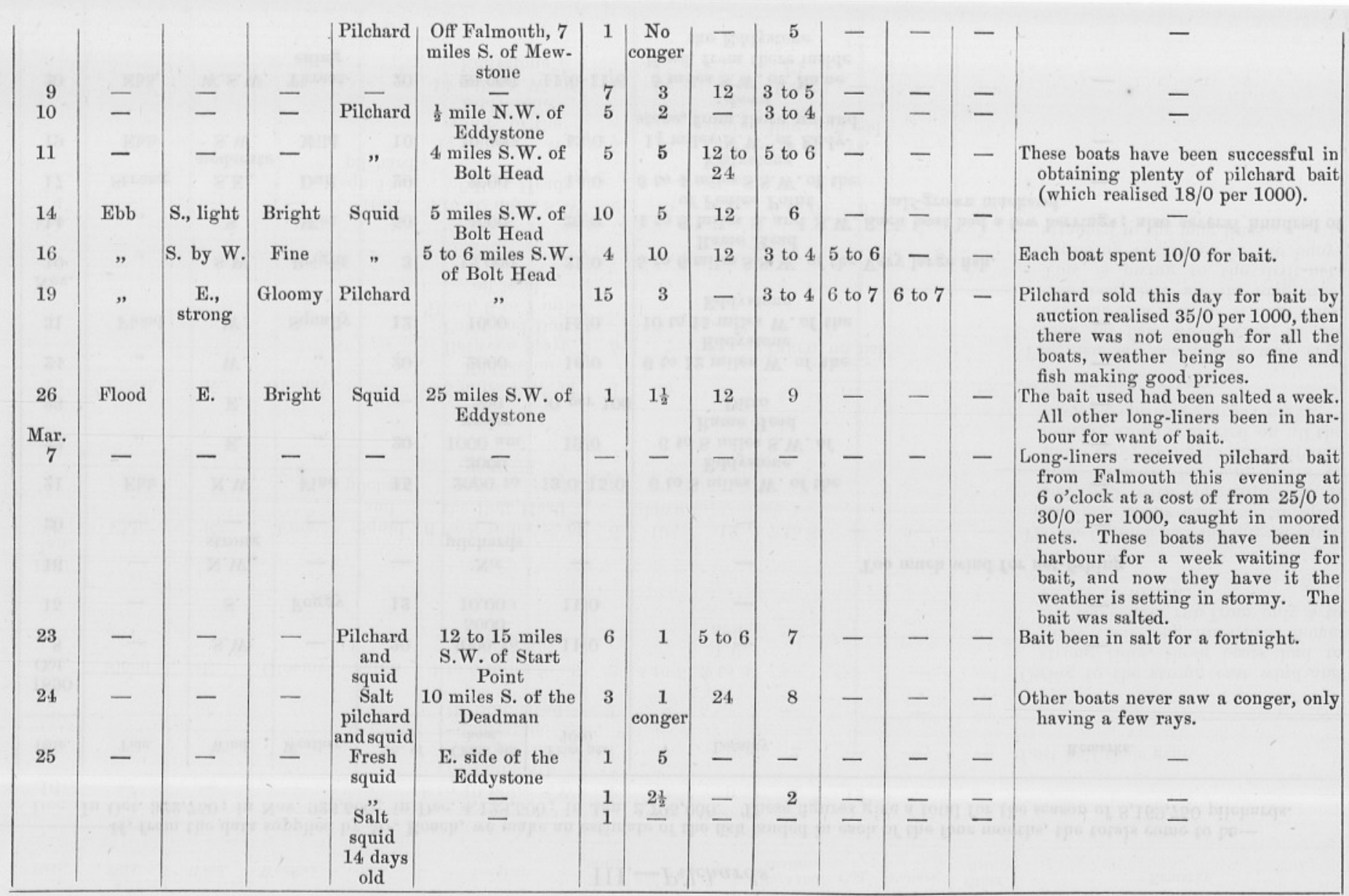


If, from the data supplied by $\mathrm{Mr}$. Roach, we make an estimate of the fish landed in each of the four months, the totals come to be-

In Oct. 322,750; in Nov. 923,500; in Dec. 4,128,500; in Jan. 2,795,000. These figures give a total for the season of 8,169,750 pilchards.

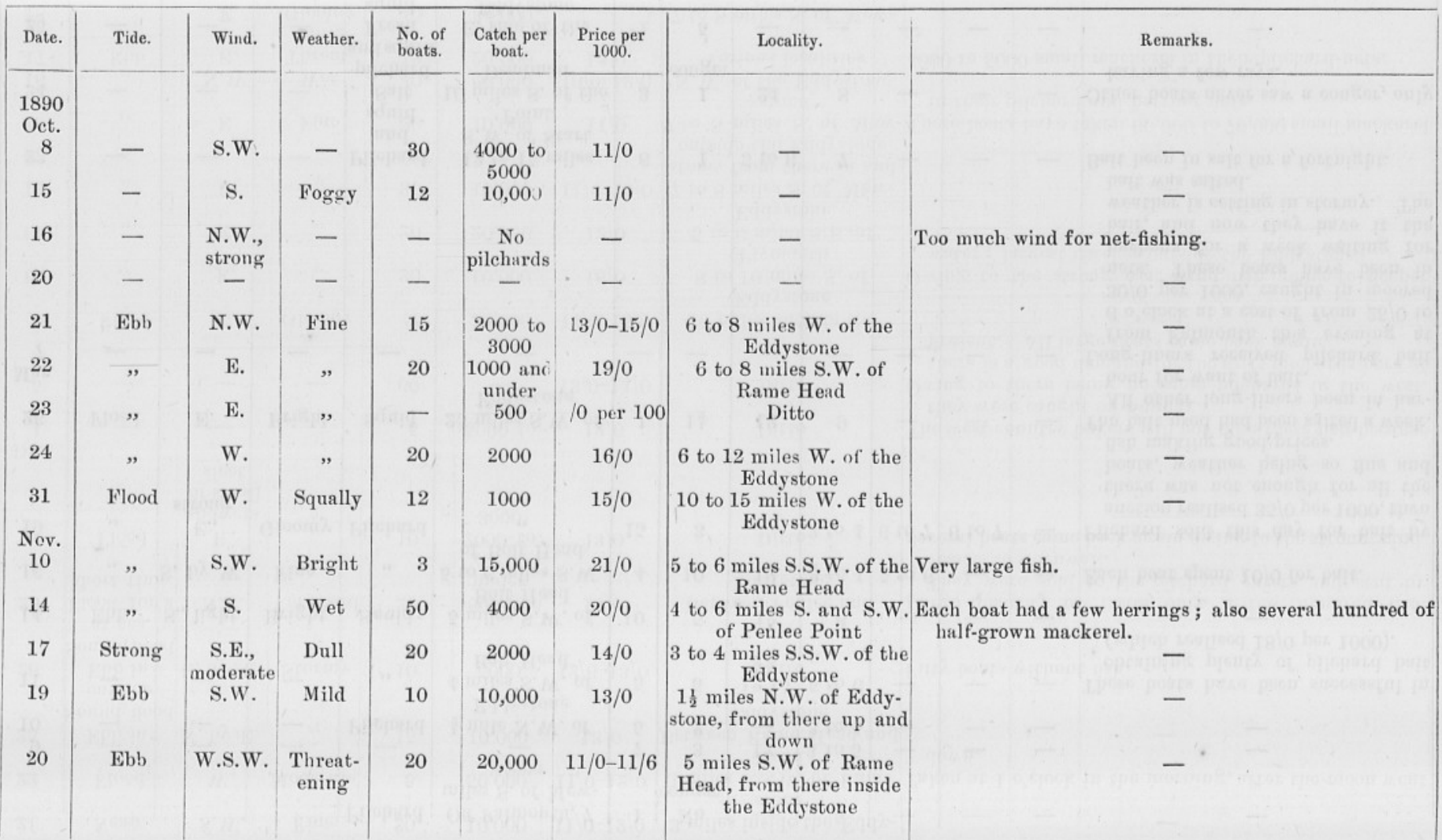




\begin{tabular}{|c|c|c|c|c|c|c|c|c|}
\hline 21 & Neap & S.W. & Fine & 20 & 10,000 & $11 / 0-12 / 0$ & $\begin{array}{l}2 \text { miles inside the Eddy- } \\
\text { stone }\end{array}$ & \\
\hline 22 & Flood & W. & Moderate & 5 & 50,000 & $11 / 0-12 / 0$ & $\begin{array}{l}3 \text { miles S.S.W. of Rame } \\
\text { Head }\end{array}$ & $\begin{array}{l}\text { Taken at } 1 \text { o'clock in the morning, after the moon went } \\
\text { down. }\end{array}$ \\
\hline 25 & $\begin{array}{c}\text { Ebb in } \\
\text { Sound, flood }\end{array}$ & N. by E. & "' & 15 & 10,000 & $13 / 0$ & $\begin{array}{l}\text { Between Rame Head and } \\
\text { Eddystone }\end{array}$ & 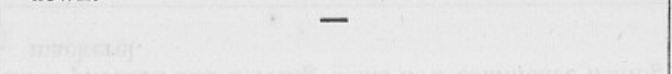 \\
\hline 26 & $\begin{array}{l}\text { outside } \\
\text { Ebb in } \\
\text { Sound, flood } \\
\text { at sea }\end{array}$ & E. by N. & Stormy & 10 & 5000 & $12 / 6-13 / 0$ & Ditto & Fifty boats without fish. \\
\hline 28 & $\begin{array}{l}\text { Flood for a } \\
\text { short time }\end{array}$ & N.E. & Very cold & 20 & $\begin{array}{l}5000 \text { and } \\
\text { under }\end{array}$ & $13 / 0$ & $\begin{array}{l}\text { Between Penlee and } \\
\text { Eddystone }\end{array}$ & $\begin{array}{l}\text { Large quantity of boats only a few hundred fish. } \\
\text { They were sold to the pilchard curers, and sent by } \\
\text { vessels to Cornwall. }\end{array}$ \\
\hline 29 & Flood & $\begin{array}{l}\text { E., } \\
\text { showers } \\
\text { of small } \\
\quad \text { sleet }\end{array}$ & " & 10 & $\begin{array}{c}2000 \text { to } \\
3000\end{array}$ & $13 / 0$ & Ditto & Several boats came back again owing to the strong wind. \\
\hline $\begin{array}{c}\text { Dec. } \\
1\end{array}$ & - & - & - & 5 & $\begin{array}{c}5000 \text { to } \\
6000\end{array}$ & $12 / 0$ & Ditto & $\begin{array}{l}\text { The west country buyers will not take these fish because } \\
\text { they were caught on Sunday. }\end{array}$ \\
\hline 5 & - & - & - & 60 & - & $13 / 0-14 / 0$ & Ditto & $\begin{array}{l}\text { Owing to there being no seine pilchards in the west, } \\
\text { there is a good demand for the drift pilchards here at } \\
\text { present. All large boats came back again. }\end{array}$ \\
\hline 9 & Flood & E. & Gloomy & 7 & 30,000 & $14 / 0-15 / 0$ & $\begin{array}{l}10 \text { miles outside the } \\
\text { Eddystone }\end{array}$ & . J f \\
\hline 10 & & E. & ", & 20 & 10,000 & $16 / 0$ & 8 to 10 miles $\mathrm{S}$. of & $\begin{array}{l}\text { Owing to the strong east wind fish going to deeper } \\
\text { water; largest catches outside the Eddystone. }\end{array}$ \\
\hline 11 & , & F. & ", & 20 & 20,000 & $12 / 0$ & 5 to 6 miles S.E. of & - \\
\hline 12 & , & E. & ," & 30 & 10,000 & $11 / 6-12 / 0$ & $\begin{array}{l}7 \text { to } 8 \text { miles } \mathrm{S} \text {. of Mew- } \\
\text { stone, from there in and } \\
\text { outside the Eddystone }\end{array}$ & - \\
\hline 13 & ", & E. & Fine & 20 & 10,000 & $11 / 0$ & 7 to 8 miles S. of Mew- & $\begin{array}{l}\text { These boats have taken } 60,000 \text { to } 70,000 \text { small mackerel } \\
\text { in their pilchard and herring nets. }\end{array}$ \\
\hline $\begin{array}{l}16 \\
17\end{array}$ & Ebb & $\begin{array}{l}\text { N.W. } \\
\text { E. }\end{array}$ & $\begin{array}{c}\text { Wet } \\
\text { Threat- }\end{array}$ & $\begin{array}{l}40 \\
12\end{array}$ & $\begin{array}{l}20,000 \\
20,000\end{array}$ & $\begin{array}{c}10 / 0-12 / 0 \\
12 / 0\end{array}$ & $\begin{array}{l}\text { N.W. of the Eddystone } \\
\text { Various localities }\end{array}$ & 4000 to 5000 small mackerel in their pilchard-nets. \\
\hline 18 & ," & E. & $\begin{array}{l}\text { ening } \\
\text { Gloomy }\end{array}$ & 40 & 30,000 & - & $\begin{array}{l}7 \text { to } 8 \text { miles S. of Mew- } \\
\text { stone, from there } S \text {. } \\
\text { and } W .\end{array}$ & F \\
\hline 23 & $\begin{array}{l}\text { Slack water } \\
\text { outside }\end{array}$ & E. & Fine & 10 & 10,000 & $12 / 0$ & N.E. of Eddystone & - \\
\hline
\end{tabular}




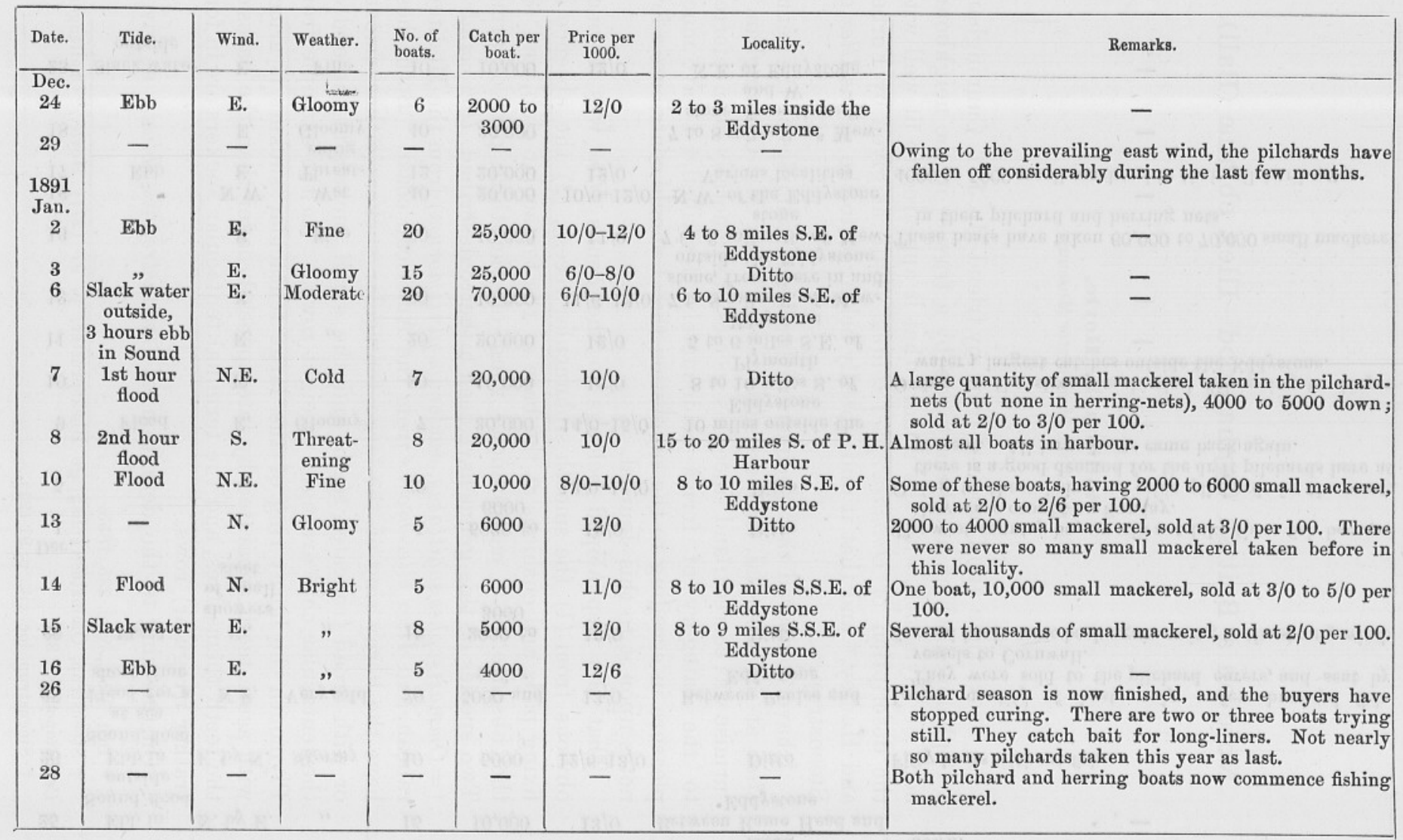

